\title{
Another motivation for the hyperbolic plane: Segments moving on the line
}

\author{
Marcos Salvai* \\ FAMAF - CIEM, Ciudad Universitaria, 5000 Córdoba, Argentina. \\ E-mail: salvai@mate.uncor.edu
}

\begin{abstract}
We give another motivation for the two dimensional hyperbolic geometry, presented through a concrete example of a surface which is not included in a canonical manner in the Euclidean space: The set of all nontrivial bounded and closed intervals in the real line, endowed with a metric which does not discriminate size.
\end{abstract}

\section{Introduction.}

An interval on the real line is said to be nontrivial if it has more than one point. Let us denote

$\mathcal{I}=\{$ nontrivial bounded and closed intervals of the real line $\}$.

Notice that $\mathcal{I}$ is not a subset on a Euclidean space (although its elements, the intervals, are so). We ask ourselves about how to define a notion of distance and of best path joining two elements of $\mathcal{I}$. The first answer which comes into our mind is the following: For $x<y$ and $x^{\prime}<y^{\prime}$ we can take

$$
\operatorname{dist}\left([x, y],\left[x^{\prime}, y^{\prime}\right]\right)=\sqrt{\left(x^{\prime}-x\right)^{2}+\left(y^{\prime}-y\right)^{2}},
$$

that is, copying the Euclidean distance from the open set $\left\{(x, y) \in \mathbb{R}^{2} \mid x<y\right\}$ by means of the obvious bijection. The best path joining these two intervals is then the curve $\gamma:[0,1] \rightarrow \mathcal{I}$ given by $\gamma(t)=\left[(1-t) x+t x^{\prime},(1-t) y+t y^{\prime}\right]$.

*Partially supported by Conicet, Secyt-UNC, Foncyt and Antorchas. 
Now, this notion of distance may be not the most convenient one if we consider the nature of the elements of $\mathcal{I}$. In a certain sense, this notion of distance discriminates the size of the segments. For instance,

$$
\operatorname{dist}\left(\left[0,10^{3}\right],\left[1,1+10^{3}\right]\right)=\sqrt{2}=\operatorname{dist}\left(\left[0,10^{-3}\right],\left[1,1+10^{-3}\right]\right) .
$$

Nevertheless, considering the relative size of the intervals, the first two of them seem to be much closer from each other than the last two of them are (let us imagine that we ask the interval $\left[0,10^{-3}\right]$ to move to the position of $\left[1,1+10^{-3}\right]$; it will seem to it very far away). In other words, they deserve a notion of distance different from the given above, accounting for this fact. We will deal with that subject below, after recalling briefly some basic facts about the hyperbolic plane. For this subject we refer the reader to [1].

\section{The hyperbolic plane.}

Let $\mathcal{H}=\left\{(u, v) \in \mathbb{R}^{2} \mid v>0\right\}$. The hyperbolic length of a differentiable curve $\gamma:[a, b] \rightarrow \mathcal{H}, \gamma(t)=(u(t), v(t))$, is defined by $\int_{a}^{b}\left\|\gamma^{\prime}(t)\right\| d t$, where

$$
\left\|\gamma^{\prime}(t)\right\|=\left|\left(u^{\prime}(t), v^{\prime}(t)\right)\right| / v(t)
$$

is the hyperbolic speed of $\gamma$ at the instant $t$ (here $|(x, y)|=\sqrt{x^{2}+y^{2}}$ denotes the Euclidean norm). This is a model for the upper half plane of LobachevskyPoincaré, the classical example of a space where the fifth axiom of Euclid does not hold: Given a line and a point outside it there exists exactly one line which does not intersect the first one. By a line in $\mathcal{H}$ we understand the image of a maximal geodesic, that is, a constant speed curve in $\mathcal{H}$ defined on the whole real line which minimizes the (hyperbolic) length between any two of its points. They are well-known to be the curves obtained by intersecting $\mathcal{H}$ with vertical (Euclidean) straight lines and with circles centered at points on the horizontal axis.

The importance of the hyperbolic plane stems also from the fact, among many other things, that it is essentially the unique complete simply connected surface with constant negative curvature.

\section{A metric on $\mathcal{I}$ which does not discriminate size.}

Recall from the introduction that we look for a notion of distance travelled by a segment moving (and changing its size as well) in the real line, and in doing that we want to be fair with the shorter intervals. 
We identify a segment in $\mathcal{I}$ by its middle point $a$ and its length $\ell$, that is, we parametrize $\mathcal{I}$ via

$$
\phi: \mathcal{A}=\left\{(a, \ell) \in \mathbb{R}^{2} \mid \ell>0\right\} \rightarrow \mathcal{I}, \quad \phi(a, \ell)=[a-\ell / 2, a+\ell / 2] .
$$

Let $\gamma(t)=\left[a_{t}-\ell_{t} / 2, a_{t}+\ell_{t} / 2\right]$ be a curve in $\mathcal{I}$ and let $\alpha(t)=\phi^{-1}(\gamma(t))=$ $\left(a_{t}, \ell_{t}\right)$ be the corresponding curve in $\mathcal{A}$. Now we see that the hyperbolic speed (1) of $\alpha$ (or equivalently of $\gamma$ ) at the instant $t$, that is,

$$
\left\|\alpha^{\prime}(t)\right\|=\left|\left(a_{t}^{\prime}, \ell_{t}^{\prime}\right)\right| / \ell_{t}
$$

is appropriate if we do not want to discriminate size, since it relativizes the Euclidean speed to the size of the interval at the instant $t$.

In the following examples we observe that, at the infinitesimal level, each segment of $\mathcal{I}$ takes itself as the yardstick to measure the size of the neighboring segments and the distance from them.

Example 1. If an interval moves to the right $s$ times its own length without changing size, then its motion will be of $s$ units if we consider the hyperbolic metric. In fact, for the curve $\gamma(t)=[a-\ell / 2, a+\ell / 2]+t \ell$, with $0 \leq t \leq s$, we have that the speed of the associated curve $\alpha(t)=(a+t \ell, \ell)$ in $\mathcal{H}$, with respect to the hyperbolic metric, is

$$
\left\|\frac{d}{d t}(a+t \ell, \ell)\right\|=\|(\ell, 0)\|_{(a+t \ell, \ell)}=|(\ell, 0)| / \ell=1 .
$$

Hence the length of $\gamma$ is $\int_{0}^{s} d t=s$. More concretely, a segment of length 1000 which moves 3000 units to the right travels the same distance as a segment which measures one thousandth and moves three thousandths in the same direction. (By the travelled distance we understand the length of the trajectory, which may be larger than the distance between the end points.)

Example 2. Let $\gamma$ be the curve in $\mathcal{I}$ defined by $\gamma(t)=\left[-e^{t}, e^{t}\right]$ and let $\alpha(t)=\left(0,2 e^{t}\right)$ be the associated curve in $\mathcal{H}$. We have that the Euclidean speed at the instant $t,\left|\alpha^{\prime}(t)\right|=\left|\left(0,2 e^{t}\right)\right|=2 e^{t}$, coincides with the size of the interval at $t$. Since the segment perceives itself as being the yardstick at any time, we are not surprised that $\gamma$ has constant (hyperbolic) speed equal to one: $\left\|\alpha^{\prime}(t)\right\|=\left|\left(0,2 e^{t}\right)\right| / 2 e^{t}=1$.

\section{Geodesics in $\mathcal{I}$.}

Since the metric we are considering on $\mathcal{I}$ is copied through the function $\phi$ from that of the hyperbolic plane, for which the vertical straight lines are trajectories of geodesics, it turns out that the curve $\gamma$ of Example 2 is a geodesic in $\mathcal{I}$. 
We also observe, considering the other geodesics of the hyperbolic plane, that in particular the trajectory of the best path in $\mathcal{I}$ joining two segments of the same length, say $\ell$, consists of segments of length larger that $\ell$. This can be explained by noticing that since each segment takes its own length as the standard to measure travelled distances or changes in its size, a bigger segment will cover great distances more easily. That is why it is not convenient for it simply moving to its final position conserving the size constant, as it would be the case for the Euclidean metric on $\mathcal{I}$, but making an extra effort at the beginning and increase its size as it moves, so it will perceive distances as shorter compared with its size, and finally, making the effort of reducing its length as it is approaching its destination.

\section{Isometries of $\mathcal{I}$.}

It is well-known that the orientation preserving isometries of the hyperbolic plane $\mathcal{H}$ are the Möbius transformations of the complex plane which preserve $\mathcal{H}$. Some of them are the mappings $f_{b}(z)=z+b$ and $g_{c}(z)=c z$, with $b, c \in \mathbb{R}$, $c>0$. The corresponding isometries of $\mathcal{I}$, induced by the identification $\phi$, which we denote with capital letters, are the following. The mapping $F_{b}$ takes each segment of $\mathcal{I}$ to the segment of equal length with initial point moved in $b$ units. As we could have expected, after that transformation the segments do not notice any change when observing around them the neighboring segments. The same happens for $G_{c}$ : The sizes of the segments and their relative positions all change in the same proportion $c$.

\section{Higher dimensions.}

If one considers balls or spheres in $\mathbb{R}^{n}$, one obtains in the same way models for the hyperbolic space of dimension $n+1$, by identifying the ball or sphere of center $a \in \mathbb{R}^{n}$ and diameter $d$ with the point $(a, d)$ in the upper half space of $\mathbb{R}^{n+1}$.

\section{References}

[1] Anderson, James W., Hyperbolic geometry, Springer Undergraduate Mathematics Series. London (2005). 